\title{
Qualities of Watermelon Juice during Shelf-life Yu-bin $\mathrm{WANG}^{1}$, Yue $\mathrm{MA}^{2}$, Xiao-yan $\mathrm{ZHAO}^{3}$ and Chao ZHANG ${ }^{4, *}$ \\ ${ }^{1}$ Beijing Vegetable Research Center, Beijing Academy of Agriculture and Forestry Sciences; \\ ${ }^{2}$ Beijing Key Laboratory of Fruits and Vegetable Storage and Processing; ${ }^{3}$ Key Laboratory of Biology and Genetic Improvement of Horticultural Crops (North China), Ministry of Agriculture; \\ ${ }^{4}$ Key Laboratory of Urban Agriculture (North), Ministry of Agriculture, 50 Zhanghua Road, Haidian District, Beijing, China \\ ${ }^{*}$ Corresponding author
}

Keywords: Watermelon, Aldehyde, 1-Nonanal, Ultra high temperature, Aroma

\begin{abstract}
The watermelon juice was treated by the ultra-high temperature and pasteurization, respectively. The quality and aroma of the pasteurized juice were evaluated during the storage at $37^{\circ} \mathrm{C}$ for $6 \mathrm{~d}$. The pasteurization was effective to maintain the total microbial counts of the watermelon juice within the limits for $6 \mathrm{~d}$. Altogether 30 volatile compounds were identified in the unpasteurized juice with the aldehyde $(70.6 \%)$ as the main component. The typical volatiles of the ultra-high temperature were reduced from $52.1 \%$ to $29.4 \%$ during the storage.
\end{abstract}

\section{Introduction}

Watermelon juice is sensitive to the heat, oxygen, light, ion, and etc. [1-4]. Various processing methods such as the high pressure treatment gives less adverse effects on aroma and color of the watermelon juice $[2,3]$; the high pressure carbon dioxide treatment holds the original properties by inactivating the polyphenoloxidase, peroxidase, and pectin methylesterase [5]; the pulsed electric field treatment holds the ((Z)-6-nonenal, 1-nonanol and (Z)-3-nonen-1-ol) content of the watermelon juice for $21 \mathrm{~d}$ [1] and maintains the antioxidant capacity [6]respectively. However, the mentioned techniques were all involved in intermittent production with the high running cost and limited production capacity.

Similarly, Ultra-high temperature (UHT) treatment inactivates spoiling micro-organisms [7], enhances the stability of the orange juice during the storage at $10{ }^{\circ} \mathrm{C}$ [7], and maintains the phenolics content of apricot nectars [8]. However, the qualities of the watermelon juice pasteurized by UHT had not been evaluated during the storage.

Therefore, the aim of the present study is to evaluate the effectiveness of UHT and Pasteurization on the qualities and aroma of the juice watermelon juice was stored at $37{ }^{\circ} \mathrm{C}$ for 1 week.

\section{Material and Methods}

\section{Processing of the Watermelon Juice}

Mature watermelon (Citrullus lanatus var. Jingxin No.3) was purchased from a local fruit market. The fruits were round with regular stripes and weighted about $3 \sim 4 \mathrm{~kg}$ per fruit. The flesh of the fruits was red with a soluble solid content of $11.5 \sim 13.5 \%$ (SSC 
was measured using a refractometer (PAL- $\alpha$, ATAGO, Japan) calibrated with distilled water).

The fruits were peeled and squeezed in a juicer (make: Philips) after stored at $4{ }^{\circ} \mathrm{C}$ for $24 \mathrm{~h}$ (HR1861, Philips Co. Beijing, China). The juice was mixed with complex food additive including arboxymethylcellulose sodium, ascorbic acid, xanthan gum, ethylene diamine tetraacetic acid, carminum, sodium pyrophosphate and etc [9]. The mixture was fully stirred and adjusted to the $\mathrm{pH} 4.1$ with the citric acid ( $1 \mathrm{~mol} / \mathrm{L})$, and adjusted soluble solid content to 8.0 with the high fructose corn syrup (4502504-01, Fresh Juice Industry (Kunshan) Co. Ltd.). The formulated juice was homogenized at $50 \mathrm{MPa}$ (NS101L2K, GEA, Parma, Italy). The juice was pasteurized by Pasteurization and UHT respectively. Specifically, the Pasteurization treatment heated the juice at $60{ }^{\circ} \mathrm{C}$ for $30 \mathrm{~min}$, and the UHT treatment heated the juice at $135^{\circ} \mathrm{C}$ for $2 \mathrm{~s}$.

The pasteurized juice was stored at $37{ }^{\circ} \mathrm{C}$ for $6 \mathrm{~d}$. The qualities and aroma of the UHT was evaluated at $1^{\text {st }}, 3^{\text {rd }}$, and $6^{\text {th }} \mathrm{d}$, which nominated as the UHT1, UHT3, and UHT6. The juice before pasteurization was considered as the Unpasteurized.

\section{Total Flora Counts}

Juice was serially diluted, plated in total count agar for total flora counts. The plates were incubated at $30{ }^{\circ} \mathrm{C}$ for $48 \mathrm{~h}$ and counted manually.

\section{Electronic Nose Analysis}

The aroma of the watermelon juice was compared by the electronic nose (PEN2, Airsense Analytics $\mathrm{GmbH}$, Schwerin, Germany). The sample of $2 \mathrm{~mL}$ was put in the testing tube. And then the electronic sensor was put into the testing tube to collect the results for $60 \mathrm{~s}$.

\section{GC-MS Analysis and Identification of Volatiles}

The volatiles of the sample were performed on an Agilent 6890 gas chromatograph coupled to an Agilent 5973I mass selective detector (Agilent Technologies, Palo Alto, CA). The volatile compounds were separated on a DB-Wax column $(30 \mathrm{~m} \times 0.25 \mathrm{~mm}$ i.d., $0.25 \mu \mathrm{m}$ film thickness, Agilent Technologies).

Volatiles were identified by comparison of their mass spectra and retention times with those of authentic standards, or by comparison of Kovats' retention indexes and mass spectrum. The K.I.s were calculated from the retention times of C6-C40 n-alkanes followed the recently method.[10]

\section{Statistical Analysis}

Analysis of variance (ANOVA) was used to compare mean differences of the results. If the differences in mean existed, multiple comparisons were performed using Duncan's Multiple Range Test. All analysis were conducted using SPSS for Window Version 19. All experiments were done in triplicates or more.

\section{Results and Discussion}

\section{Total Microbial Counts of Watermelon Juice}

The effect of storage time on total microbial counts of the watermelon juice is shown in Fig. 1. The initial count of the Pasteurization and UHT was lower than $2.0 \mathrm{Log}$ CFU/mL. Subsequently, the total microbial count of each treatment was enhanced with the storage time. The total microbial count of the Pasteurization and UHT reached 2.6 and 5.6 Log CFU/mL, respectively, on the $6^{\text {th }}$ d. Therefore, the 
Pasteurization was effective to maintain the microbial count of the watermelon juice.

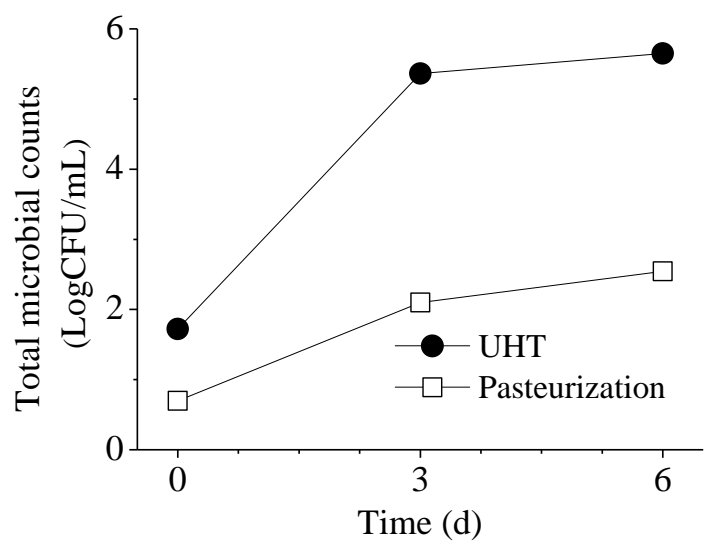

Figure 1 Effect of storage time on total microbial counts of the watermelon juice

\section{Aroma of the Watermelon Juice}

The pasteurized juice was stored at $37{ }^{\circ} \mathrm{C}$ and the aroma of the pasteurized juice was compared by the sensor array of 10 electrodes in the electric nose during the storage (Fig. 2). The dimensions of electrical responds were reduced by the principal component analysis. The main component 1 and 2 contributed $93.43 \%$ and $4.17 \%$ for the total watermelon aroma, respectively. The main component 1 represented the main aroma of the watermelon juice. The area of the Unpasteurized had no overlap with the stored watermelon juice. Consequently, the aroma of the UHT1, UHT3, and UHT6 was different to that of the unpasteurized juice. Interestingly, the aroma of the berry juice pasteurized by the UHT is well maintained [11], while the heating at $90{ }^{\circ} \mathrm{C}$ for $28 \mathrm{~s}$ leads to a significant aroma change of the apple cider [12].

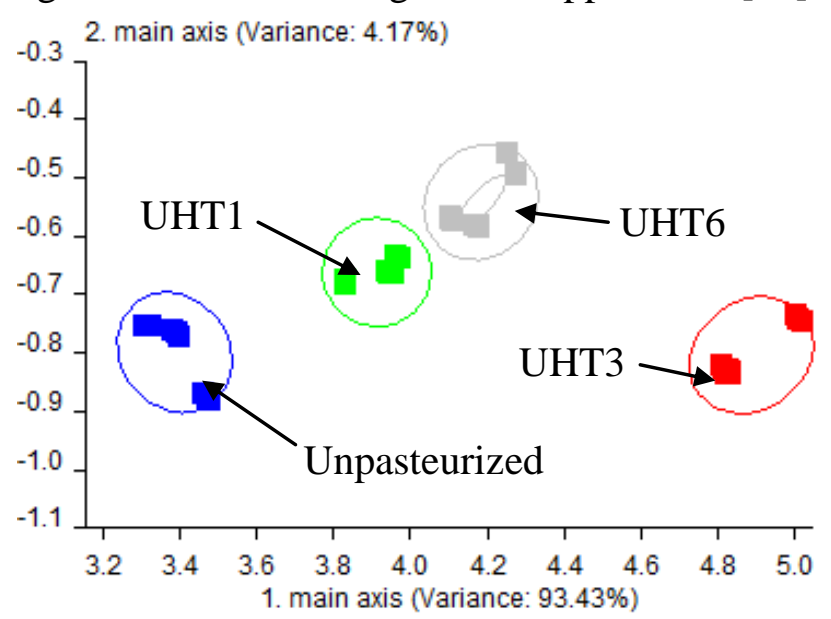

Figure 2 Effect of storage time on aroma of the watermelon juice

\section{Volatiles of the Watermelon Juice}

Total 30, 28, 25, and 28 volatiles were tentatively assigned in the unpasteurized, UHT1, UHT3 and UHT6, respectively (Table 1). The aldehyde, alcohol, ketone, alkane, and ester constituted $70.6 \%, 15.8 \%, 9.24 \%, 4.42 \%$, and $0.26 \%$, respectively, in the unpasteurized watermelon juice. The acid was not found in the unpasteurized watermelon juice. Consequently, the aldehyde was the main component in unpasteurized watermelon juice. 
Table 1. Volatile contents $(\%)$ of the watermelon juice during storage

\begin{tabular}{|c|c|c|c|c|c|}
\hline Type & Volatile & Unpasteurized & UHT1 & UHT3 & UHT6 \\
\hline \multirow{3}{*}{ 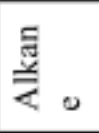 } & Tetradecane & 0.65 & $-*$ & - & - \\
\hline & 2-Pentylfuran & 3.08 & 5.52 & - & $=$ \\
\hline & 1-Hexadecene & 0.69 & 1.05 & - & - \\
\hline \multirow{22}{*}{$\begin{array}{l}\overrightarrow{0} \\
\frac{0}{0} \\
\frac{0}{4}\end{array}$} & Subtotal & 4.42 & 6.57 & 0 & 0 \\
\hline & 1-Nonanol & - & $=$ & - & 3.81 \\
\hline & (3Z)-3-Nonen-1-ol & 3.42 & 2.3 & 1.73 & 0.72 \\
\hline & (E)-2-Nonen-1-ol & 4.31 & 3.07 & 2.4 & 1.9 \\
\hline & (3E,6Z)-3,6-Nonadien-1-ol & $=$ & $=$ & 2.5 & 1.95 \\
\hline & Phenethyl alcohol & 3.06 & 4.04 & 5.23 & 3.98 \\
\hline & 2-Methyl-1-propanol & 0.99 & - & - & - \\
\hline & Hexyl alcohol & 0.93 & 0.18 & 2.53 & 1.1 \\
\hline & 3-Methyl-1-butanol & 0.19 & - & 3.64 & - \\
\hline & 2-Ethylhexanol & 0.37 & - & 1.56 & - \\
\hline & 2-Octanol & 1.04 & $=$ & - & $=$ \\
\hline & Capryl alcohol & - & - & - & 2.2 \\
\hline & Geraniol & 0.25 & - & - & - \\
\hline & 2-Hexyloctanol & - & 0.69 & - & $=$ \\
\hline & 1-Octen-3-ol & - & - & 1.49 & 3.25 \\
\hline & Dodecenol & - & 0.33 & - & - \\
\hline & (2E)-2-Undecen-1-ol & $=$ & - & - & 0.25 \\
\hline & Dodecyl alcohol & 0.47 & - & - & - \\
\hline & 1-Tridecanol & 0.8 & 2.77 & 2.02 & 1.43 \\
\hline & 1-Pentadecanol & - & 0.57 & - & - \\
\hline & 1-Hexadecanol & - & 0.53 & - & - \\
\hline & 1-Nonadecanol & - & 0.3 & - & - \\
\hline & Subtotal & 15.83 & 14.78 & 23.1 & 20.59 \\
\hline & 1-Nonanal & 8.45 & 9.18 & 6.85 & 2.81 \\
\hline & (2E)-2-Nonenal & 10.1 & 9.22 & 8.56 & 7.35 \\
\hline & (E,Z)-2,6-nonadienal & 25.8 & 22.59 & 18.7 & 10.9 \\
\hline & 2-Hexenal(E) & 4.32 & 4.21 & 2.93 & 1.05 \\
\hline & Octanal & 5.21 & 3.22 & - & - \\
\hline ¿ & (E)-2-Octenal & 0.83 & $=$ & - & 0.15 \\
\hline 민 & (E)-Hept-2-enal & 1.23 & - & - & - \\
\hline & Decyl aldehyde & 6.08 & 5.87 & - & - \\
\hline & 2-Undecenal & - & $=$ & 5.13 & 8.25 \\
\hline & (E)-2-Dodecenal & 7.65 & 6.07 & - & - \\
\hline & Tetradecanal & - & 0.66 & 0.51 & 0.49 \\
\hline & Pentadecanal & 0.58 & 0.89 & 1.03 & $=$ \\
\hline & Subtotal & 70.25 & 61.91 & 43.71 & 31 \\
\hline & 3-Hydroxy-2-butanone & 1.47 & - & - & - \\
\hline & 6-Methyl-5-hepten-2-one & 3.97 & 2.95 & 5.74 & $=$ \\
\hline ב & 6,10-Dimethyl-5,9-undecadien-2-one & 2.83 & 5.31 & 4.32 & 3.18 \\
\hline$\stackrel{0}{a}$ & 2-Dodecanone & - & - & 1.46 & - \\
\hline & 2-Pentadecanone & 0.33 & 2.46 & 3.9 & $=$ \\
\hline & 2-Nonadecanone & 0.64 & - & - & - \\
\hline & Subtotal & 9.24 & 10.72 & 15.42 & 3.18 \\
\hline & Nonanoic acid & - & - & 4.85 & 10.01 \\
\hline & Acetic acid & - & - & 1.68 & 4.09 \\
\hline$\frac{\pi}{0}$ & 2-Methyl butyric acid & -- & - & - & 8.97 \\
\hline & Hexanoic acid & - & $=$ & 2.52 & 6.31 \\
\hline & Octanoic acid & - & - & 3.23 & 7.51 \\
\hline
\end{tabular}


Table 1, cont. Volatile contents (\%) of the watermelon juice during storage

\begin{tabular}{|c|c|c|c|c|c|}
\hline \multicolumn{2}{|r|}{ Subtotal } & 0 & 0 & 12.28 & 36.89 \\
\hline \multirow{7}{*}{ 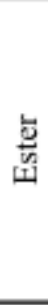 } & Isopropyl palmitate & - & 0.46 & - & - \\
\hline & Dodecyl Acetate & - & - & - & 0.16 \\
\hline & Diisobutyl phthalate & 0.26 & 2.51 & 5.49 & 4.48 \\
\hline & Dimethyl phthalate & - & - & - & 2.67 \\
\hline & Octyl Formate & $=$ & 0.22 & - & $=$ \\
\hline & Heptyl forMate & - & - & - & 0.9 \\
\hline & Allyl hexanoate & - & 2.83 & - & 0.13 \\
\hline & Subtotal & 0.26 & 6.02 & 5.49 & 8.34 \\
\hline
\end{tabular}

*: not detected.

Remarkably, the aldehyde content of the unpasteurized, UHT1, UHT3, and UHT6 was $70.6 \%, 61.9 \%, 43.7 \%$, and $31.0 \%$, respectively. The aldehyde was believed to be the main aroma of the watermelon juice. The aldehyde content reduced significantly during the storage, while the alcohol, acid, and ester content of the juice were enhanced during the storage.



Figure 3 Effect of storage on typical volatiles of the watermelon juice

The C9 alcohol and aldehyde are the key aroma of the watermelon juice. The 1-Nonanol, (E)-2-Nonen-1-ol, (3Z)-3-Nonen-1-ol, (3E,6Z)-3,6-Nonadien-1-ol, 1-Nonanal, (E,Z)-2,6-Nonadienal, and (2E)-2-Nonenal were designated as the typical volatiles of the watermelon in the watermelon juice. The content of the typical volatiles of the unpasteurized, UHT1, UHT3, and UHT6 was $52.1 \%, 46.4 \%, 40.7 \%$, and $29.4 \%$, respectively (Fig. 3). Hence, the watermelon aroma of the pasteurized watermelon juice was reduced with the storage time.

\section{Acknowledgement}

The authors are grateful to financial support of China Agriculture Research System (CARS-26), Beijing Academy of Agricultural and Forestry Sciences (KJCX20170205), and Beijing Key Laboratory of Fruits and Vegetable Storage and Processing (Z141105004414037).

\section{Reference}

[1] I. Aguiló-Aguayo, M. Montero-Calderón, R. Soliva-Fortuny, O. Martín-Belloso, Changes on flavor compounds throughout cold storage of watermelon juice processed by high-intensity pulsed electric fields or heat, J. Food Eng. 100 (2010) 43-49. 
[2] Q. Liu, R.-F. Wang, B.-B. Zhang, X.-Y. Zhao, D. Wang, C. Zhang, Protein secondary structure changes of watermelon juice treated with high hydrostatic pressure by FTIR spectroscopy, J. Food Process Eng. 37 (2014) 543-549.

[3] C. Zhang, B. Trierweiler, W. Li, P. Butz, Y. Xu, E. C. Rüfer, Y. Ma, X. Zhao, Comparison of thermal, ultraviolet-c, and high pressure treatments on quality parameters of watermelon juice, Food Chem. 126 (2011) 254-260.

[4] M. Feng, K. Ghafoor, B. Seo, K. Yang, J. Park, Effects of ultraviolet-C treatment in Teflon ${ }^{\circledR}$-coil on microbial populations and physico-chemical characteristics of watermelon juice, Innov. Food Sci. Emerg. Technol. 19 (2013) 133-139.

[5] Y. Liu, X. Hu, X. Zhao, H. Song, Combined effect of high pressure carbon dioxide and mild heat treatment on overall quality parameters of watermelon juice, Innov. Food Sci. Emerg. Technol. 13 (2012) 112-119.

[6] G. Oms-Oliu, I. Odriozola-Serrano, R. Soliva-Fortuny, O. Martin-Belloso, Effects of high-intensity pulsed electric field processing conditions on lycopene, vitamin C and antioxidant capacity of watermelon juice, Food Chem. 115 (2009) 1312-1319.

[7] B. K. Tiwari, C. P. O' Donnell, K. Muthukumarappan, P. J. Cullen, Ascorbic acid degradation kinetics of sonicated orange juice during storage and comparison with thermally pasteurised juice, LWT Food Science and Technol. 42 (2009) 700-704.

[8] W. Huang, X. Bi, X. Zhang, X. Liao, X. Hu, J. Wu, Comparative study of enzymes, phenolics, carotenoids and color of apricot nectars treated by high hydrostatic pressure and high temperature short time, Innov. Food Sci. Emerg. Technol. 18 (2013) 74-82.

[9] Y. B. Wang, Y. Ma, X. Zhao, C. Zhang, Qualities and aroma of watermelon juice during storage at $4 \mathrm{oC}$, Advances in Biological Sciences Research (ABSR), volume 4.

[10]X. Tang, H. He, Y. Liu, X. Zhao, Identification of aroma compounds in watermelon juice by SPME-GCMS, Acta Hort. 944 (2012) 183-191.

[11]I. Dalmadi, K. Polyak-Feher, J. Farkas, Effects of pressure-and thermal-pasteurization on volatiles of some berry fruits, High Pressure Res. 27 (2007) 169-171.

[12] G. G. Rye, D. G. Mercer, Changes in headspace volatile attributes of apple cider resulting from thermal processing and storage, Food Res. Int. 36 (2003) 167-174. 